\title{
Association of Parenteral Nutrition Catheter Sepsis with Urinary Tract Infections*
}

\author{
Debra S. Kovacevich, R.N., B.S.N.,+ Walter C. Faubion, R.N., M.S., +

 \\ From the $\dagger$ Parenteral and Enteral Nutrition Team, $\ddagger$ Department of Infectious Disease, Department of Surgery and Medicine, School of \\ Pharmacy, University of Michigan Medical Center, Ann Arbor, Michigan
}

\begin{abstract}
The infection rate (IR) of central venous catheters (CVCs) used for parenteral nutrition (PN) was prospectively evaluated over a 12 -month period, with emphasis on the relationship between CVC sepsis and preexisting sites of infection. Sepsis was presumed if the CVC tip or blood culture was positive, or if defervescence followed CVC removal. Four hundred seventy-three CVCs were placed for PN for a total of 5,422 patient days, with a mean length of stay of 11.5 days. Twenty two CVCs led to sepsis for an IR of $4.65 \%$ or 4.06 infections per 1000 patient days. Twenty of the 22 septic CVCs were in patients with other sites of infection. The IR was $12.0 \%$
\end{abstract}

$(20 / 166)$ when other sites of infection were present and $0.65 \%$ $(2 / 307)$ in the absence of a second site. The mean length of stay was 13.2 days for CVCs with other sites of infection and 10.3 days for CVCs with no other site of infection. Fifty nine percent of septic CVCs had secondary sites of infection that included urinary tract infections (UTI). Of all septic CVCs, $22.7 \%$ had no site of infection other than L"TI. The presence of UTI appears to present a high risk of CVC sepsis. Appropriate identification and treatment of UTI prior to CVC insertion is recommended. (Journal of Parenteral and Enteral Nutrition 10:639-641, 1986)
Infection remains the major complication of the placement of central venous catheters for parenteral nutrition (PN). Specialized teams have had a significant impact in lowering sepsis rates. With the increase in number of patients receiving parenteral nutrition, especially those who are critically ill, it has become exceedingly important to recognize those individuals who may be at risk for catheter sepsis. Identification of individuals with specific risks allows practitioners to develop guidelines and protocols for more effective treatment and prevention of catheter sepsis.

There is little information, however, about the correlation between catheter-related sepsis and other existing sites of infection. This prospective study was undertaken with the goal of discovering patterns that may exist between catheter sepsis rates and other sites of clinical infection.

\section{MATERIALS AND METHODS}

All central venous catheters (CVCs) used for paren. teral nutrition were studied prospectively during a 12 month period. An infected CVC was defined at the outset of the study as that giving rise to a positive, semiquantitative CVC tip culture, ${ }^{1}$ or a positive blood culture drawn through the catheter, or the combination of a negative CVC tip but immediate defervescence upon removal. Any presumed CVC sepsis was evaluated by the Department of Infectious Disease for confirmation. Each

Received for publication. December 19, 1985.

Accepted for publication. April 1, 1986

Reprint requests: Debra S. hovacevich, R.N. B.S.N., Parenteral and Enteral Nutrition Team, Lniversity of Michigan Medical Center. 1500 E. Medical Center Drive/Box 0008. Ann Artur. MII 4\$109-0008.

* Presented at the Ninth Clinical Congre. $A-1$ 'F . Miami. Florida, January 21-24, 1985 .
CVC placed was evaluated prospectively regarding patient location, demographics, if the catheter was rethreaded by the use of a guidewire, physician placing the catheter, date placed and removed, CVC site location, catheter longevity, and reason for removal. An assessment also was made as to whether the catheter placement and care were according to guidelines as established by the Center for Disease Control. ${ }^{2}$ As in many institutions, the use of a PN catheter for any purpose other than PN was discouraged. Violations included using the line for blood drawing, blood infusion, medication administration, fluids for hydration, or central venous pressure readings. Catheters which were rethreaded over a guide wire were also included in this category. In addition, existing sites of infection were recorded along with the causative bacteria. Included in this category were cultures from wounds, sputum. drains, peripheral blood, or blood drawn through the catheter, urine catheters, and the CVC catheter tip. The white blood cell count, total protein, and albumin were recorded along with other laboratory parameters to determine risk facturs.

All CVC dressing changes were done by the $-t$ aff nurses according to guidelines set up by the University of Michigan Parenteral and Fnteral Vutrition Team using a specially designed kit. The gauze method of dressing change was utilized and was performed every $4 \mathrm{ith}$ for catheters placed in the subclavian vein and every $24 \mathrm{hr}$ for those placed in the internal jugular vein. Each patient with a CVC was seen three times a week by nurses on the team to ensure that the dressings were done according to the protocol established.

Central venous catheters usually were placed by surgical house officers with supervision by senior staff. Aseptic technique was maintained during central catheter insertion by first cleansing the area for 2 minutes 
with a povidine-iodine scrub and allowing it to dry. The use of a mask and sterile gloves was mandatory. The use of a sterile gown was encouraged but not required.

The Desert Intracath, which is made of polyvinylchloride, was the catheter most used in the study. It was recommended that it be sutured lateral to the insertion site on the chest wall in order to maintain an occlusive dressing. Other catheters used were the Cordis catheter and the proximal port of the Swan Ganz catheter by Edwards. Luer lock extension tubing was used in all patients and was changed at the same time the dressing was changed. An inline filter manufactured by Cutter was mandatory with all parenteral nutrition therapy and was changed daily with the intravenous tubing.

Statistical analysis was done using the chi-square method.

\section{RESULTS}

A total of 473 catheters were studied. This represented a total of 5422 patient days with a mean CVC longevity of 11.5 days. Of the $473 \mathrm{CVCs}$ placed for parenteral nutrition, 22 led to sepsis for an infection rate of $4.65 \%$ or 4.06 infections per 1000 patient days with a mean CVC longevity of 13.2 days.

All 22 septic CVCs had a positive tip culture. No catheter was identified with a negative tip culture with defervescence upon removal. Of the 22 catheters with a positive tip culture, 14 grew the identical organism on both the tip and blood. Conversely, three catheters had organisms growing on the tip that were different from those in the blood. Five septic CVCs had a positive tip culture and negative blood culture. Pathogens isolated from the intravascular lines included Serratia marcescens (three cases), Candida species (five cases), Staphylococcus epidermidis (five cases), Escherichia coli (one case), Enterococcus (one case), and mixed bacterial organisms (two cases).

Factors which were not associated with an increased risk of CVC sepsis were age $(p=0.2)$, hypoalbuminemia defined as an albumin of less than $2.8 \mathrm{~g} / \mathrm{dl},(p=0.02)$, and hypoproteinemia defined as a total protein of less than $5.5 \mathrm{~g} / \mathrm{dl}(p=0.14)$.

Factors which were associated with an increased risk of catheter sepsis included lack of adherence to protocol, the presence of a secondary site of infection, and the presence of a urinary tract infection.

Protocols were strictly followed in a total of 325 catheters studied (Table I). Ten of these 325 CVCs became infected for an infection rate of $3.0 \%$. There were 148 nonprotocol CVCs of which 12 became septic, leading to an infection rate of $8.1 \%$, a statistically significant increase over the protocol group.

TABLE I

CVC sepsis and protocol adherence

\begin{tabular}{lllll}
\hline & \multicolumn{4}{c}{ CVC sepsis } \\
\cline { 2 - 5 } & & Yes & No & Total \\
\hline Protocol & Yes & 10 & 315 & 325 \\
Adherence & No & 12 & 136 & 148 \\
& Total & 22 & 451 & 473 \\
\hline
\end{tabular}

One hundred sixty-six catheters were placed in patients who had a secondary site of infection (Table II). Of these 166, 20 became infected, leading to an infection rate of $12 \%$ in this group with a mean catheter longevity of 13.2 days. The remaining 307 catheters were in patients that had no other sites of infection. Two of these catheters became infected for an infection rate of $0.65 \%$ with a catheter longevity of 10.3 days.

We then focused on the association of urinary tract infections (UTI) and catheter sepsis (Table III). A total of 429 catheters were placed in patients who did not have a UTI, of which nine became infected for an infection rate of $2.1 \%$. This contrasted with a total of 35 catheters placed in patients with a UTI, 13 of which became infected for an infection rate of $22.7 \%$. Of these 13 catheters, five had the same causative bacteria infecting both the catheter and the urine.

Further analysis was done in patients with CVC sepsis and a UTI $v$ s those with other secondary sites of infection (Table IV). A total of 131 catheters were placed in patients who had a known site of infection other than the urinary tract. Seven of the catheters became infected for a rate of $5.3 \%$.

\section{DISCUSSION}

Catheter-related sepsis continues to be the most frequent complication of central parenteral nutrition therapy. Septicemia rates, associated with the administration of intravenous feedings, have been reported as high as $93 \%$ and as low as $14 \%$ between the years 1969 and $1970 .^{3-5}$ With the formation of nutritional support teams to develop insertion and catheter care protocols, CVC sepsis has decreased dramatically. This decrease was

TABLE II

CVC sepsis and secondary sites of infections

\begin{tabular}{llrrr}
\hline & \multicolumn{4}{c}{ CVC sepsis } \\
\cline { 2 - 5 } & & Yes & No & Total \\
\hline Secondary & Yes & 20 & 146 & 166 \\
Infection & No & 2 & 305 & 307 \\
& Total & 22 & 451 & 473 \\
\hline
\end{tabular}

TABLE III

CVC sepsis and presence of a urinary tract infection

\begin{tabular}{rlrrr}
\hline & \multicolumn{4}{c}{ CVC sepsis } \\
\cline { 2 - 5 } & & Yes & No & Total \\
\hline \multirow{3}{*}{ UTI } & Yes & 13 & 22 & 35 \\
UTI & No & 9 & 429 & 438 \\
& Total & 22 & 451 & 473 \\
\hline
\end{tabular}

TABLE IV

CVC sepsis and urinary tract infections vs other secondary sites

\begin{tabular}{llrrr}
\hline & \multicolumn{4}{c}{ CVC sepsis } \\
\cline { 2 - 5 } & & Yes & No & Total \\
\hline UTI & Yes & 13 & 22 & 35 \\
Other infection & Yes & 7 & 124 & 131 \\
& Total & 20 & 146 & 166 \\
\hline
\end{tabular}


shown in a study by Nehme ${ }^{6}$ in which those patients receiving $P N$ were randomized into two groups. Those followed by their nutritional support team had a $1.7 c_{c}$ sepsis rate, whereas those treated by their respective physician had an infection rate of $11 \%$. Similarly, Sanders and Sheldon' reported a $20 \%$ infection rate before the initiation of a team, with a reduction to $8 \%$ when one was developed.

Similar to these other institutions, we have also developed a multidisciplinary Parenteral and Enteral Nutrition Team with responsibility for the care and development of protocols for patients receiving parenteral nutrition. These protocols are reviewed and revised on a yearly basis. Inservices on the dressing change procedure and PN protocols are given 20 times per year by the nurses on the team to newly employed nurses.

Nonadherence to developed protocols in CVCs used for $\mathrm{PN}$ was usually an active decision on the part of the house officer treating the patient. The most frequent reason for violation was limited venous access. Removal of the CVC for suspected sepsis was also up to the discretion of the house officer. Our findings confirmed those of many other institutions in which central catheters not used exclusively for parenteral nutrition had an increased risk of septicemia. ${ }^{8,9}$ It is the belief of these authors that infection is due to a breakdown in aseptic technique while performing CVP readings, medication administration, blood administration, blood drawing, and piggybacking fluids for hydration. These procedures require manipulations of the intravascular line with the use of stopcocks, needles, and syringes several times within $24 \mathrm{hr}$.

The patients in this study were not clustered on one specific unit, but were distributed among 21 general care and intensive care units. As discussed in the results, there was a lack of any common organisms cultured with only three catheters positive for $S$. aureus. It is therefore highly unlikely that any cross-contamination occurred between the patients studied and medical staff as described by Knittle. ${ }^{10}$

Abel and associates ${ }^{11}$ documented in a careful prospective study that $75 \%$ of catheters removed for suspected sepsis were unnecessarily discontinued. Evaluation of these patients revealed in all of them a septic focus other than the catheter. Dillion and associates ${ }^{12}$ found all patients with catheter sepsis had at least one infection elsewhere, whereas $55.3 \%$ of the nonseptic patients (with catheters) had at least one infected site.

In circumstances in which other prostheses were placed, similar results were encountered. Quinzer et al ${ }^{13}$ studied the infection rates of intracardiac prosthetic valves. In $29.2 \%$ of their patients, the genitourinary tract or oral cavity was the probable source of the infecting microorganism causing endocarditis. Urinary tract infections were implicated in $12.5 \%$ of all patients. with an overall infection rate of $4.4 \%$. In yet another study involving septic prosthetic heart valves, $7.8 \%$ of the patients studied had either a urinary tract infection or had undergone genitourinary manipulation prior to the onset of bactremia. ${ }^{i+1}$

Of even greater interest is the work done in the evaluation of total hip replacements and secondary sites of infection. A 7 -y study was done by Wroblewski and Del Sel $^{15}$ in which all male patients who developed urinary retention and required catheterization following arthroplasty were evaluated. The overall deep sepsis rate of the implants was $6.2^{c} \mathrm{C}$ in these patients. In addition. Irvine et al $^{16}$ studied the efficiency of sterile air flow systems in the operating room $u s$ the use of prophylactic antibiotics, and determined that the organisms cultured from patients with deep sepsis were those found in the urine in $60 \%$ of cases.

The data from this study demonstrate an association between CVC sepsis and urinary tract infections. Based on our data and what has been learned from studies with artificial prostheses, we recommend that any patient being considered for CVC placement with a known or suspected urinary tract infection be evaluated for UTI. If a UTI is present, the patient should be placed on an appropriate antimicrobic for $24 \mathrm{hr}$ before CVC insertion. We believe this approach will provide a further adjunct to the prevention of CVC sepsis.

\section{REFERENCES}

1. Maki DG, Wise CF, Sarafin HWP: A semiquantitative culture method for identifying intravenous catheter-related infection. $\mathrm{N}$ Engl J Med 296:1305-1309, 1977

2. Simmons BP: Guidelines for prevention of intravascular infections. Hospital Infection Control 28J-29P, February 1982

3. Boeckman CR, Krill CE: Bacterial and fungal infections complicating parenteral alimentation in infants and children. $J$ Pediatr Surg 5:117-126, 1970

4. Ashcraft KW, Leape LL: Candida sepsis complicating parenteral feeding. JAMA 212:454-456, 1970

5. Dudrick SJ, Groff DB, Wilmore DW: Long-term venous catheterization in infants. Surg Gynecol Obstet 129:805-808, 1969

6. Nehme AE: Nutritional support of the hospitalized patient. JAMA 243:1906-1908, 1980

7. Sanders RA, Sheldon GF: Septic complications of tot al parenteral nutrition. Am J Surg 132:214-220, 1976

8. Curry CR, Quie PG: Fungal septicemia in patient- receiving parenteral hyperalimentation. N Engl J Med 285:1221 $12: 12: 1971$

9. Syndman DR, Murray SA, Kornfeld SJ, et al: Total parenteral nutrition-related infections. Am I Med 73:695-699, 198:

10. Knittle MA, Eitzman DV, Baer J: Role of hand contamination of personnel in epidemiology of gram-negative nosocomial infections. J Pediatr 86:433-437, 1975

11. Ryan A, Abel M. Abbott WM ex al: (atherer complications in

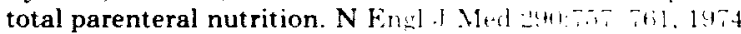

12. Dillon JD, Schaffner $W$, Van Wa ( $W$, et al: Septicemia and total parenteral nutrition. JA.11 2:3:1341 1:34. 197:3

13. Quinzer RW, Fdward I.I), Levin \&: A comprarative -tudy of 48

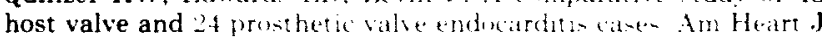
92:15-22. $19: 5$

14. Dismuke: WF. Karchmer AW. Buckles Prosthetic valve endocardit.. analysis of 38 cases. Circul....... , ....j5-377, 1973

15. Wroblew iki BM. del Sel HJ: Lrethral instrumentation and deep sepsis in total hip replacement. Clin Orthop 146:209-212, 1980

16. Irvine R. Johnson BL, Amstutz HC: The relationship of genitourinary tract procedures and deep sepsis after total hip replacements. Surg Gynecol Obstet 139:701-706, 1974 